\title{
Lesson of the month 2: Dry skin, yellow nails and breathlessness
}

\author{
Authors: Janet Fallon, ${ }^{\mathrm{A}}$ Shuja Majeed, ${ }^{\mathrm{B}}$ Huzaifa Adamali ${ }^{\mathrm{C}}$ and Harsha Gunawardena ${ }^{\mathrm{D}}$
}

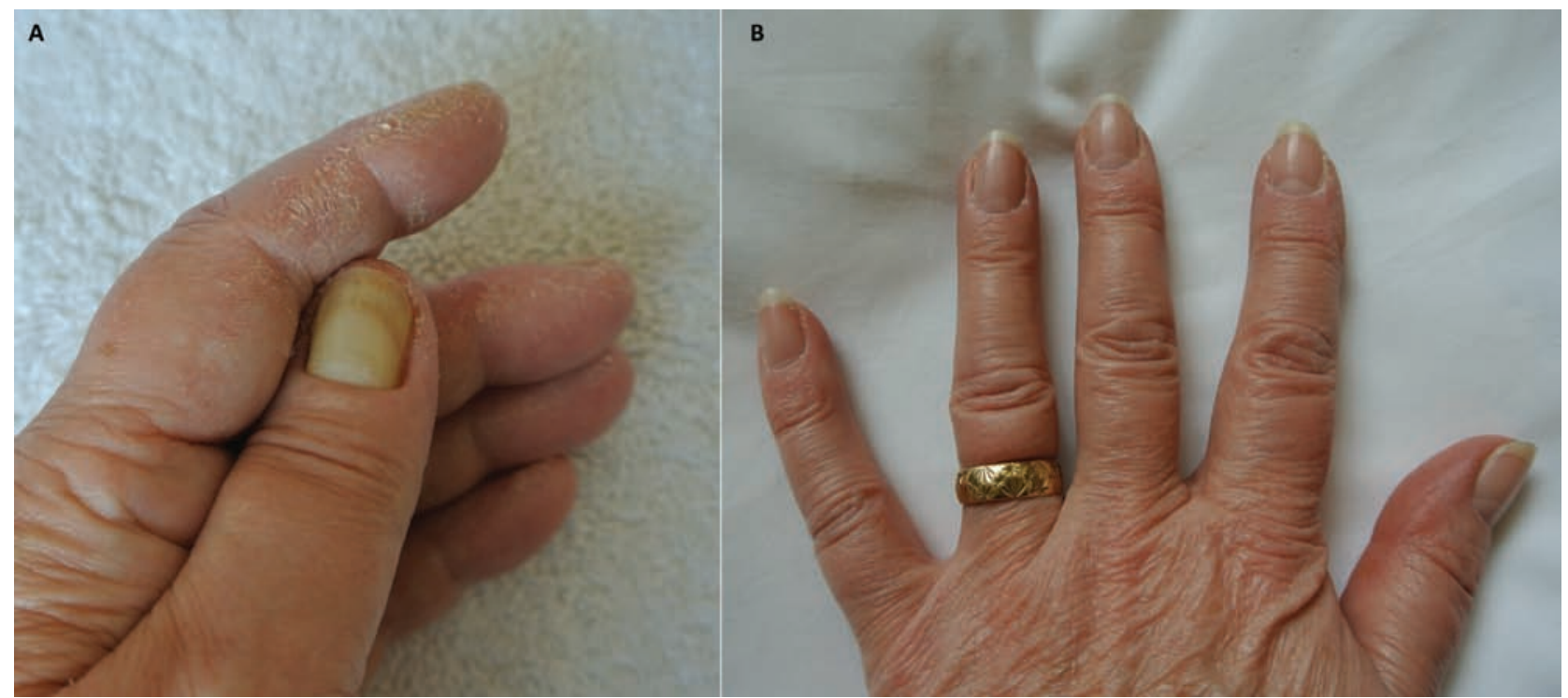

Fig 1. Nail and hand changes in interstitial lung disease. A - yellowing and thickening of the nails and cracking of the skin along the lateral aspects of the index, middle and ring fingers; $\mathrm{B}$ - improvements in symptoms following treatment.

Interstitial lung disease (ILD) is a common clinical problem, representing a group of diseases consisting of inflammation and progressive fibrosis of the lung. In some cases, an underlying cause is not identified; however, a significant proportion of ILD is associated with connective tissue disease (CTD). A detailed history and examination is the most important part of the assessment of patients with suspected ILD and will direct further investigation. This case illustrates the importance of identifying the symptoms and signs of CTD when assessing a patient with ILD. In addition, we describe an unusual presenting manifestation of yellow nails, which is not a recognised feature of CTD-ILD, but improved following immunomodulatory treatment for the overall condition.

KEYWORDS: Autoimmune connective tissue disease, interstitial lung disease

Author: ${ }^{A}$ respiratory specialist registrar, Southmead Hospital,

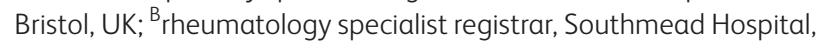
Bristol, UK; C ${ }^{C}$ consultant respiratory physician, Southmead Hospital, Bristol, UK; ${ }^{\mathrm{D}}$ consultant rheumatologist and honorary lecturer, Southmead Hospital, Bristol, UK

\section{Case history}

A 59-year-old female patient was referred to a general respiratory clinic with a history of productive cough and breathlessness. She had previously presented to her GP with symptoms of chest tightness ongoing for a few months. A chest X-ray performed in the community showed patchy inflammatory changes at the left middle and lower zones.

She had a background of chronic sinusitis requiring previous sinus surgery but had no other past medical history. She was not on any regular medication. She gave an additional history of symmetrical swelling of the fingers, elbows and wrists, together with intermittent myalgia.

Clinical examination revealed yellow and thickened nails, with marked cracking of the skin of the fingers (Fig 1A). Chest examination revealed fine inspiratory crackles at the lung bases. Oxygen saturations were $96 \%$ at rest but dropped to $92 \%$ after 150 meters of corridor walking. Lung function tests showed a modest reduction in static lung volumes and gas transfer; forced vital capacity (FVC) 2.6 (98\%), forced expiratory volume in 1 second (FEV1) 2.0 (91\%), FEV1/FVC ratio 78\%, residual volume (RV) 1.5 (84\%), total lung capacity (TLC) 3.9 (83\%), transfer factor for carbon monoxide (TLCO) 6.2 (84\%), KCO 1.5 (95\%). A high resolution computerised tomography 

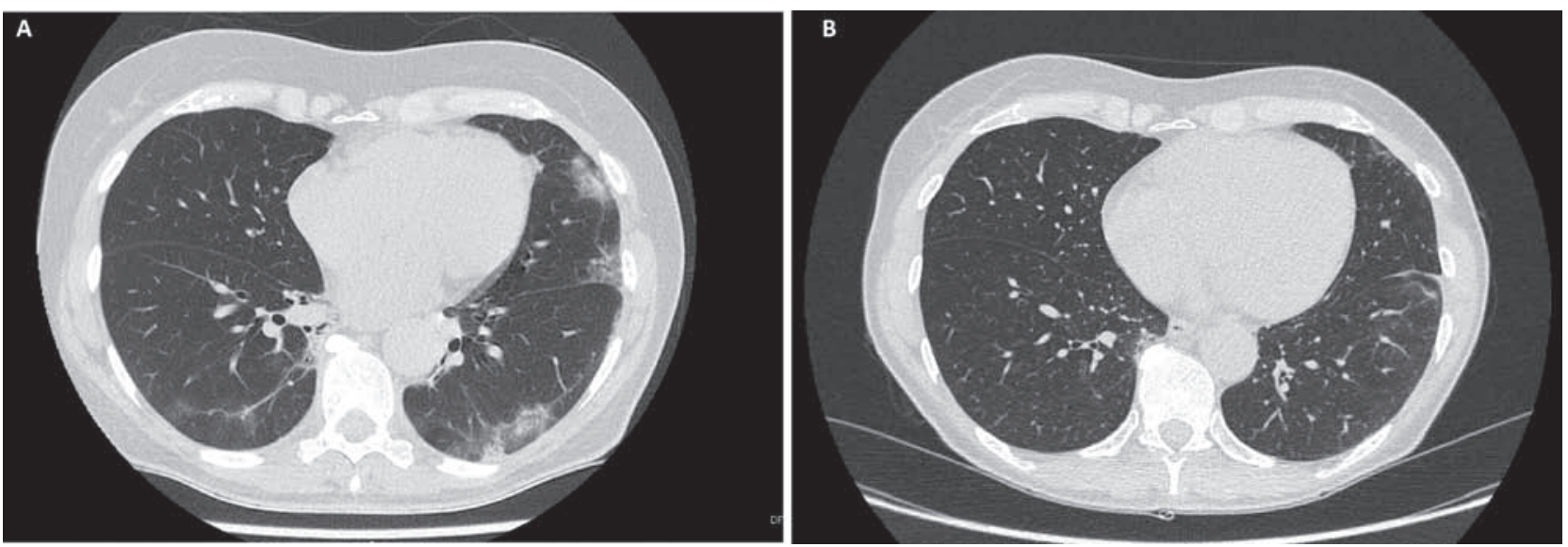

Fig 2. High resolution computerised tomography images of the chest in interstitial lung disease. A - bilateral subpleural infiltrates and reticulation; $B$ - resolution of subpleural infiltrates and reticulation following treatment.

(HRCT) scan of the chest showed bilateral subpleural patchy consolidation (Fig 2A). Anti-nuclear antibody (ANA) was negative but HEp-2 testing demonstrated cytoplasmic staining and subsequent extended screening confirmed anti-Jo1 antibodies.

This presentation is consistent with a diagnosis of antisynthetase syndrome characterised by the presence of anti-tRNA synthetase antibodies, namely anti-Jol, and specific clinical manifestations. The predominant feature in this case was interstitial lung disease (ILD) with cutaneous features, including unusually yellow nails.

The patient was treated with pulsed intravenous methylprednisolone, followed by prednisolone and mycophenolate. She also received topical steroid therapy for the skin changes. These treatments resulted in improvements in the respiratory and systemic symptoms, resolution of the skin and nail changes (Fig 1B) and radiographic improvement on follow-up CT imaging (Fig 2B). Lung function testing also revealed an increase in lung volumes and gas transfer: FVC 3.1 (116\%), FEV1 2.2 (96\%), FEV1/FVC ratio 70\%, RV 1.8 (98\%), TLC 4.8 (99\%), TLCO 8.6 (116\%), KCO 1.93 (124\%).

\section{Discussion}

Antisynthetase syndrome is an overlap autoimmune connective tissue disease associated with distinct clinical manifestations, including myositis, ILD, arthritis, fever, Raynaud's phenomenon and skin changes in the hands - termed mechanic's hands. ${ }^{1}$ The latter is a pathonomonic feature of hyperkeratotic, fissured skin on the palmar and lateral aspects of the fingers, typically the thumb, index and middle fingers. Occasionally, these changes result in irregular, dirty-appearing horizontal lines that resemble those of a manual labourer. ${ }^{2}$ As far as the authors are aware, there are no documented cases of antisynthetase syndrome presenting with yellow nails.

In terms of lung involvement, this condition is most commonly associated with a non-specific interstitial pneumonia, characterised by ground glass opacities in a basal and peripheral distribution. Patients can also show patterns of ILD consistent with organising pneumonia. ${ }^{3}$ Changes can progress to a fibrotic phase.

The principle treatment approach in antisynthetase syndrome with ILD usually consists of steroids in conjunction with additional immunomodulatory agents such as cyclophosphamide, azathioprine and mycophenolate. In cases refractory to conventional immunosuppression, rituximab has been shown to be effective in connective tissue disease-related ILD. ${ }^{4}$ -

\section{Conflicts of interest}

The authors have no conflicts of interest to declare.

\section{Acknowledgements}

Written consent was obtained from the patient to publish the clinical details and images in this article.

\section{References}

1 Lundberg IE, Miller FW, Tjärnlund A, Bottai M. Diagnosis and classification of idiopathic inflammatory myopathies (Review Symposium). J Intern Med 2016;280:39-51.

2 Stahl NI, Klippel JH, Decker JL. A cutaneous lesion associated with myositis. Ann Intern Med 1979;91:577.

3 Waseda Y, Johkoh T, Egashira R et al. Antisynthetase syndrome: pulmonary computed tomography findings of adult patients with antibodies to aminoacyl-tRNA synthetases. Eur J Radiol 2016;85:1421-6.

4 Sharp C, McCabe M, Dodds N et al. Rituximab in autoimmune connective tissue disease-associated interstitial lung disease. Rheumatology 2016;55:1318-24.

Address for correspondence: Dr Janet Fallon, Bristol Lung Centre, Southmead Hospital, Bristol BS16 1JE, UK.

Email: janetfallon@doctors.org.uk 